\title{
Mass Adsorbed and Surface Forces in Ternary Solution as Nonsolvent Was Added to the Point of Precipitation: Polystyrene in Mixtures of Cyclopentane and Linear Pentane
}

\author{
Harry E. Johnson, Hsuan-Wei Hu, and Steve Granick* \\ Department of Materials Science and Engineering, University of Illinois, \\ Urbana, Illinois 61801
}

Received June 1, 1990; Revised Manuscript Received September 26, 1990

\begin{abstract}
The influence of ternary solvent composition on mass adsorbed and on surface forces between adsorbed polystyrene layers was investigated. Polystyrene $\left(M_{w}=500000\right.$ and $\left.3.84 \times 10^{6}\right)$ was adsorbed onto silicon oxide and mica from dilute solution $\left(0.15 \mathrm{mg} \mathrm{mL}^{-1}\right)$ in mixtures of cyclopentane and $n$-pentane at 23 ${ }^{\circ} \mathrm{C}$. Simplicity was afforded by the fact that the two solvents were expected to mix nearly athermally. FTIR-ATR (Fourier transform infrared spectroscopy in attenuated total reflection) was used to measure mass adsorbed at the silicon oxide interface, and surface forces were measured between overlapping adsorbed layers at the muscovite mica interface. Three principal findings emerged. (1) The levels of adhesion and the thicknesses of the adsorbed polymer layers, measured in the surface force apparatus, increased significantly with the addition of nonsolvent, but there was a continuous transition between behavior in homogeneous solution and in phase-separated solution. (2) The mass of polystyrene adsorbed at the silicon oxide interface increased systematically with higher volume fraction, $\phi_{3}$, of $n$-pentane. The relation $\Delta \Gamma=\phi_{3}{ }^{1.13}(\Delta \Gamma$ is the mass adsorbed in excess of that adsorbed from undiluted cyclopentane) described this increase empirically, amounting to a factor of 2 before the point of precipitation. (3) Possibly contrary to intuition, there occurred no detectable ( $<0.1 \mathrm{mg} \mathrm{m}^{-2}$ ) depletion of the nonsolvent, $n$-pentane, from the adsorbed polymer layers (surface coverages 4 and $5.5 \mathrm{mg} \mathrm{m}^{-2}$ ). Two hypotheses are suggested; they pertain not only to polymers in ternary solution but also to polymer chains adsorbed from a single solvent. First, the adhesion in two-phase solution sets a resonable upper bound on the interfacial tension between a polymer-rich surface and a polymer-poor coexisting solution, $\gamma \approx 0.05 \mathrm{~mJ} / \mathrm{m}^{2}$. Second, the large but still microscopic amount adsorbed just before phase separation is consistent with the expectation that adsorbed monolayers should broaden to form multilayers containing chains, none of whose segments are located directly at the substrate surface.
\end{abstract}

\section{Introduction}

We report below experiments that explore the effect of solvent quality on the surface excess of solvent and of polymer and on the surface forces in a mixed-solvent system. Polystyrene (PS) was dissolved in solvent mixtures of cyclopentane (CP) and $n$-pentane up to and just beyond the point of phase separation. Simplicity was provided by the fact that the two isomeric solvents were expected to mix nearly athermally. In addition, it was expected that $n$-pentane, because of its similar chemical structure to that of cyclopentane, would be less prone to displace adsorbed polymer than would other potential nonsolvents. However, cyclopentane and $n$-pentane differ in solvent quality for PS: cyclopentane is a $\theta$ solvent near the temperature of these experiments, while $n$-pentane is a nonsolvent.

While adsorption of polymer chains from a single solvent has been studied extensively, little is known about adsorption from mixtures of solvents. Adsorption is the outcome of an interplay between considerations of solvent quality and of interactions between the substrate surface and the species in solution. Obviously single solvents are particularly simple conceptually. The complex mixedsolvent situation also deserves understanding, however, in part because it is the rule in many practical applications. Examples include those applications in which adsorbed polymers are used to protect against the flocculation of colloidal particles, as in the paint, food, and ink industries. The paint industry, for example, employs several solvents in order to control the effective solvent quality, the volatility, and the viscosity. ${ }^{1}$ Still other examples of adsorption from mixed-solvent systems relate to adsorption chromatography and to the lubrication of sliding surfaces by mixtures of base oils. Insofar as the scientific study of polymer adsorption is justified by its relevance to practical applications, it is useful to compare behavior in the pure and in the mixed-solvent situations.

Study of the bulk solution behavior of polymers dissolved in a mixture of two solvents has a long history. ${ }^{2-15}$ Polymer fractionation is a widespread and extremely useful application of mixed solvents in bulk solution. Of the two solvents, one will generally be a better solvent than the other. Therefore, in a mixture of solvent and poorer solvent, on expects intuitively that (providing the two solvents mix well) the composition of the solvent mixture within the space pervaded by the polymer molecules will be enriched in the better solvent: i.e., the polymer will preferentially adsorb the better solvent. The consequences for the resulting chain dimensions are still being explored by calculations, ${ }^{10}$ simulations, ${ }^{11}$ and experiments. ${ }^{12-14}$ Certain features remain poorly understood in detail. For example, it happens that a polymer may dissolve in a mixture of two solvents in which, separately, it would be insoluble; this synergistic effect is believed to result from unfavorable enthalpy of mixing of the two solvents. ${ }^{15}$

The inherent complexity of the physics involved is apparent when one considers the possible enthalpic interactions. Phrased in a generalized Flory-Huggins language, one has three parameters rather than a single Flory-Huggins interaction parameter $(\chi)$ to contend with: $\chi_{12}, \chi_{13}$, and $\chi_{23}$, where interactions may occur between one solvent (1), the polymer (2), and a second solvent (3).

When a polymer is adsorbed from mixed solvents, the number of parameters needed to describe the possible interactions grows still more. It is usual to describe the intensity of segment-substrate interactions in binary solution by a segment-surface interaction parameter, $\chi_{8}{ }^{16}$ or its equivalent. ${ }^{17}$ This is essentially the dimensionless free energy needed for replacement of an adsorbed solvent molecule by a chain segment. Note that $\chi_{B}$ is a relative 
free energy: polymer segments must compete with solvent molecules for sites on the surface. In ternary solution, the intensity of segment-substrate interactions therefore calls for description according to $\chi_{\mathrm{s}, 12}, \chi_{\mathrm{s}, 13}$, and $\chi_{\mathrm{s}, 23}$.

Again, despite the complexity, purposeful adsorption from mixed solvents has long been routine in separating species using adsorption chromatography. Here the solvent composition is adjusted to control the strength of adsorption. ${ }^{18-20}$ In fact, Cohen-Stuart and co-workers showed that the effective adsorption energy of a polymer segment can even be determined on this basis, by measuring the critical solution concentration of a displacer solvent to the point at which the polymer desorbs. ${ }^{20,21}$ Although differential segment-solvent interactions in solution (referred to above) were assumed to be a secondorder effect in this analysis, it is one of the few existing methods to quantify the intensity of substrate-segment interactions.

The sensitivity of force-distance profiles between adsorbed polymer layers to small differences in solvent composition, when adsorbed from mixed solvents, was suggested by our earlier work concerning polystyrene adsorbed onto muscovite mica from cyclopentane (CP) containing $5 \%$ linear pentane. ${ }^{22}$ Qualitatively different force-distance profiles were obtained when a CP of $>99 \%$ purity was used, ${ }^{23}$ as compared to when a CP containing $5 \%$ linear pentane isomers was used. ${ }^{22}$ The forces at long range above the $\theta$ temperature $\left(T_{\theta}\right)$ were systematically attractive when PS was dissolved in the former solvent and were systematically repulsive when the same PS was dissolved in the latter solvent. These differences were surprising and provided the initial impetus for the experiments below in which the mixed-solvent situation was explored purposefully.

Effects of mixed solvents were also investigated by Marra and co-workers using the surface force apparatus. ${ }^{24-26}$ Polystyrene (PS) was adsorbed from miscible tolueneheptane mixtures (toluene being a good solvent for PS and heptane being a nonsolvent). The adsorbed amount increased as the heptane content was raised from zero (no PS adsorption), to $25 \%$, and then to $50 \%{ }^{24}$ Quantification of the adsorbed amount was not possible, owing to the unknown degree to which the adsorbed layer was preferentially swollen with toluene. Forces were also measured after the dissolved PS solution was replaced, first with toluene-heptane mixtures of higher heptane conten ${ }^{24}$ and then with mixtures of acetone and heptane (both of the latter liquids being nonsolvents for PS). ${ }^{25}$ Not surprisingly, large adhesion was measured between two swollen PS layers in the presence of large amounts of nonsolvent. 24,25 In addition, it appeared that the acetone induced desorption of PS; presumably, being more polar than PS, acetone displaced PS segments from the surface. ${ }^{25}$ More recently, force-distance measurements were also reported of PS adsorbed onto mica from acetone-heptane mixtures, acetone-cyclohexane mixtures, and water-cyclohexane mixtures. These emphasized the consequences of displacement of adsorbed polymer segments by acetone and the consequences of capillary condensation by water. ${ }^{26}$

The study undertaken by us has three parts. First, measurements were made of force-distance profiles of polystyrene (PS) adsorbed onto muscovite mica from mixtures of cyclopentane and $n$-pentane. The fraction of $n$-pentane was varied from zero to beyond the point of precipitation. The temperature, $T=23^{\circ} \mathrm{C}$, was just above the $\theta$ temperature, $T_{\theta}$, of polystyrene in pure cyclopentane $\left(T_{\theta}=19.5^{\circ} \mathrm{C}^{27,28}\right)$. Next, for adsorption of PS onto a silicon oxide substrate from solvent mixtures of these
Table I Characterization of the Polystyrene Standards

\begin{tabular}{ccl}
\hline$M_{\mathrm{p}}^{\mathrm{a}}$ & $M_{\mathrm{w}} / M_{\mathrm{n}}$ & \multicolumn{1}{c}{ source } \\
\hline 490000 & $<1.08$ & Polymer Laboratories \\
515000 & $<1.07$ & Polymer Laboratories \\
575000 & $<1.06$ & Pressure Chemical \\
3840000 & 1.04 & Toyo Soda
\end{tabular}

${ }^{a}$ Manufacturer's data based on gel permeation chromatography.

same compositions, independent FTIR-ATR measurements (Fourier transform infrared spectroscopy in attenuated total reflection) were undertaken as well. Finally, FTIR-ATR measurements provided a method to determine an upper bound on the extent of solvent segregation within the adsorbed PS layers.

\section{Experimental Section}

The experimental protocol for the surface force experiments ${ }^{23}$ and the infrared experiments ${ }^{29,30}$ were essentially as described previously.

Surface Force Measurements. In the surface force experiments, two atomically smooth, step-free mica sheets were glued onto cylindrical lenses and mounted at right angles to one another using our home-built surface apparatus. The distance between the sheets were measured by multiple-beam interferometry between the backs of the sheets, and the distance-dependent force between the sheets was inferred from the deflection of a weak leaf spring. First, the sheets were brought together at a slow rate in $\mathrm{N}_{2}$ atmosphere to verify the expected jump into contact resulting from attractive van der Waals forces. Next, the presence of oscillatory structural forces between a mica surface separated by pure cyclopentane was verified. Finally filtered PS solution was added to the cyclopentane to form a dilute PS solution, and this solution was incubated for at least 1 day at large separation, at the temperature of subsequent measurements, before measurements were begun.

In the data shown in the figures, pip left denotes measurements made as the surfaces were brought together and pip right denotes measurements made as the surfaces were separated. Agreement of these measurements (after equilibration for up to $2-5 \mathrm{~min} /$ data point) indicates reversibility over the experimental time scale. The forces were not affected by the time (up to $30 \mathrm{~min}$ ) that the adsorbed layers were kept compressed before they were separated, although we have presented evidence elsewhere ${ }^{23}$ that suggests that slower bridging relaxations do occur over periods of hours. This does not affect the current study, which emphasizes surface forces exclusive of bridging.

The solvent composition was changed by withdrawing a small portion of cyclopentane from the measurement chamber and then adding the equivalent amount or more of $n$-pentane. It was verified that force-distance profiles did not change with longer incubation times than the ones we used.

Polystyrene standards were purchased and used as received. Their characteristics and sources are listed in Table I. Three fractions with $M \sim 500000$ were used because of the limited quantity of those we used first.

Cyclopentane (Eastman Kodak or Fluka; $99 \%$ pure by GLC) was stored over type $4 \mathrm{~A}$ molecular sieves and was freshly distilled before use. Protio-n-pentane (Aldrich) was stored and distilled before use in the same manner. Deuterio- $n$-pentane (Cambridge Isotope Laboratories) was used as received.

FTIR-ATR Measurements. Adsorption onto the surface of a cylindrical infrared prism was monitored by using the attenuated total reflection (ATR) infrared Circle Cell optics sold by SpectraTech, Inc. Infrared spectra were collected, and the silicon ATR prism was prepared, by using methods described previously.29,30 In brief, the silicon was prepared before each experiment with the following protocol: reflux for several hours in ethyl acetate, ultrasound in ethyl acetate for $10 \mathrm{~min}$, treat in oxygen-purged argon ion plasma for $1 \mathrm{~min}$, and then commence the experiment using freshly distilled solvents that had been dried over type $4 \mathrm{~A}$ molecular sieves. After assembling the experimental cell, the cell was filled with cyclopentane and allowed to sit for $30-60$ min; this improved the base-line stability and appeared to serve 


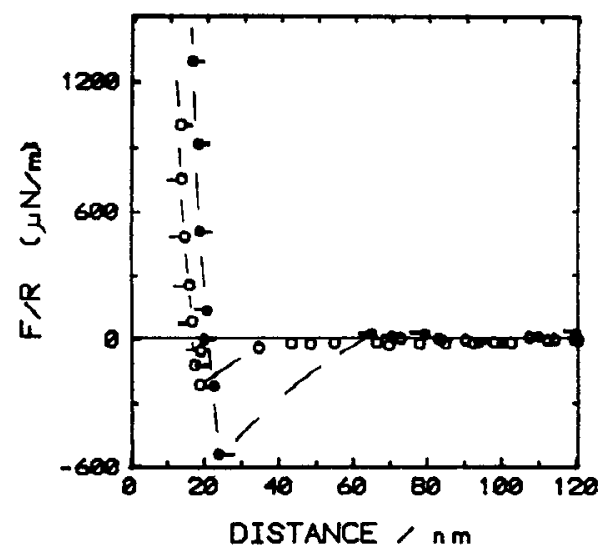

Figure 1. Influence of solvent composition on surface forces for PS $(M=515000)$ at $23^{\circ} \mathrm{C}$. Force, normalized by radius of curvature of the mica cylinders, is plotted against distance. Open circles: undiluted cyclopentane. Filled circles: volume fraction of $n$-pentane is 0.15 in the solvent mixture.

the purpose of allowing ambient airborne adsorbents to dissolve away. At this point the cyclopentane in the cell was discarded and polymer solution was added.

Under ambient conditions, such as we used, it is well-known that silicon is covered by a native oxide layer. Calibrations showed that Beer's law held to a good approximation for these thin films of low absorbance, as expected, ${ }^{32}$ and that PS free in solution contributed $<1 \%$ to the measured PS absorption peaks. The penetration depth of the evanescent wave was far larger than the characteristic thickness of the adsorbed polymer layers. The penetration depth was $d_{p}=0.28 \mu \mathrm{m}$ at $2900 \mathrm{~cm}^{-1}$ and was $d_{p}=$ $0.38 \mu \mathrm{m}$ at $2100 \mathrm{~cm}^{-1}$. Therefore, the peak intensities did not contain information concerning segment distribution as a function of depth but represented, by Beer's law, the surface excess mass of adsorbed polymer in toto.

Polymer solutions were prepared in cyclopentane and then diluted with $n$-pentane to the desired solvent composition. The volume fraction of $n$-pentane in solution was calculated by weight and density, assuming no volume change upon mixing. The surface coverage corresponding to a given absorbance intensity was calibrated from the known solution signal as described elsewhere. ${ }^{30}$

Uncertainty in the levels of surface excess was $\pm 0.1 \mathrm{mg} \mathrm{m}^{-2}$, except for experiments of many hours duration, during which period there sometimes occurred base-line drift.

\section{Results}

Influence of Solvent Composition on Surface Forces. Polystyrene $\left(M_{\mathrm{w}}=515000\right)$ was adsorbed onto mica from a solution of concentration $0.15 \mathrm{mg} \mathrm{mL}^{-1}$ in cyclopentane of $>99 \%$ purity. The temperature was 23 $\pm 0.5^{\circ} \mathrm{C}$. The polymer was allowed to incubate at large separation for at least 1 day. After measurements were made of the force-distance profile, $n$-pentane was added to the surface force apparatus as described above, $5 \%$ by volume each time. The system was equilibrated for at least $8 \mathrm{~h}$ more after adding $n$-pentane before measurements were taken. The solutions containing volume fractions of $n$-pentane equal to 0.05 and 0.10 were translucent and appeared to be homogenous, but the solutions turned cloudy and the PS began to precipitate at a volume fraction of $n$-pentane equal to 0.15 .

In Figure 1 are plotted force-distance profiles for PS in cyclopentane containing volume fractions of $n$-pentane of $\phi_{3}=0.00$ and 0.15 . In Figure 2 are plotted force-distance profiles for $\phi_{3}=0.05$ and 0.10 . Force, normalized by the mean radius of curvature of the mica surfaces, is plotted against the distance of closest separation between the mica cylinders. This normalized force is proportional, by the

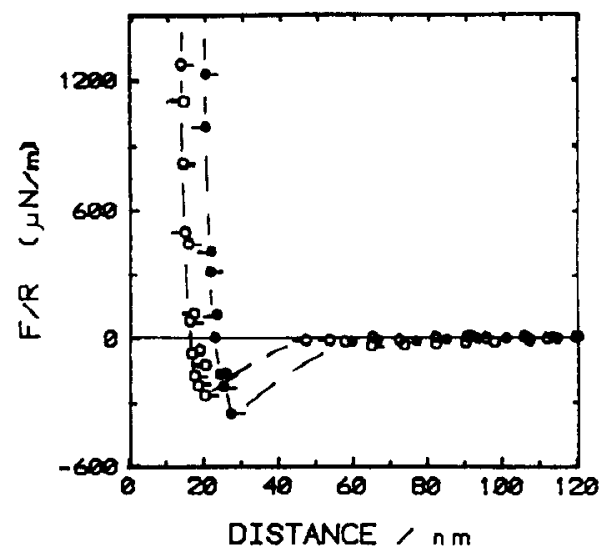

Figure 2. Influence of solvent composition on surface forces for PS $(M=515000)$ at $23^{\circ} \mathrm{C}$. Force, normalized by radius of curvature of the mica cylinders, is plotted against distance. Open circles: volume fraction of $n$-pentane is 0.05 . Shaded circles: volume fraction of $n$-pentane is 0.10 in the solvent mixture.

Derjaguin approximation, ${ }^{33}$ to the equivalent energy per unit area between parallel plates at this same distance.

These force-distance profiles look alike, but they differ in their quantitative features. At large separations, where forces between the adsorbed layers first appeared, the forces were moderate. Their magnitude increased slowly with diminishing separation, and an attractive well was observed. At short range the repulsion was steep. The depth of the attraction (referred to below as "adhesion") and the range of the forces increased systematically with an increasing proportion of $n$-pentane in the solvent mixture.

Attractive interactions were observed in every case, with or without added $n$-pentane, unlike our earlier measurements in a polystyrene-cyclopentane system in which we saw monotonic repulsion using a source of cyclopentane described (as purchased) to contain $5 \%$ linear pentane impurities. ${ }^{22}$ It seems that the repulsion observed in that study stemmed from some other impurity, not $n$-pentane.

In view of this discrepancy with earlier results, which we obtained using a different source of cyclopentane, ${ }^{22}$ the possibility of impurities was considered; however, this appeared unlikely. First, the FTIR-ATR measurements showed no detectable impurities in the spectra. Second, there was self-consistency in the present experiments between two different sources of linear pentane. Third, the present sources of cyclopentane were specified by the manufacturers to be of higher purity than our previous source. We have previously performed extensive measurements using one of these sources of cyclopentane. ${ }^{23}$ In that study, we obtained findings quantitatively consistent with the behavior measured by other workers for PS adsorbed from cyclohexane (at equal temperatures of corresponding state relative to the bulk $\theta$ temperature). ${ }^{23}$ Fourth, in any event, the cyclopentane was not a variable in these experiments; the changes we studied occured as the level of added $n$-pentane was varied. In summary, we do not find evidence for a role of impurities in these measurements.

We now proceed to further discussion of the surface force results. First we consider the thickness at the point of harsh repulsion. In Figure 3, this thickness is plotted against solvent composition at the compressions 1000 and $2000 \mu \mathrm{N} / \mathrm{m}$. The thickness at $2000 \mu \mathrm{N} / \mathrm{m}$ increased from just under $12 \mathrm{~nm}$ (undiluted CP) to $18 \mathrm{~nm}\left(\phi_{3}=0.10\right)$. Note that the point of precipitation is apparent in this graph: the compressed layer thickness was slightly less after precipitation than just before it. 


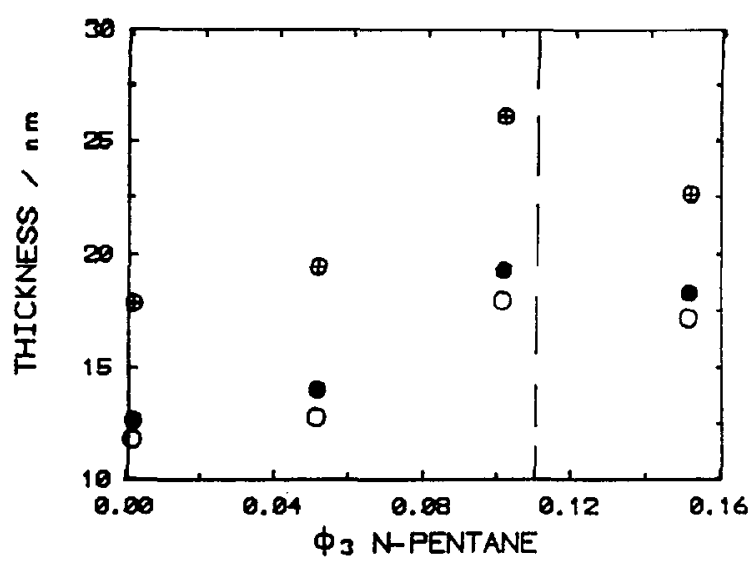

Figure 3. Thickness at maximum attractive force (crossed circles) and the compressed thickness of adsorbed PS layers at $2000 \mu \mathrm{N} / \mathrm{m}$ (open circles) and at $1000 \mu \mathrm{N} / \mathrm{m}$ (filled circles), plotted atainst the $n$-pentane volume fraction in the solvent mixture. The vertical bar denotes the region of solvent composition in which FTIR-ATR experiments showed precipitation of polymer to occur.

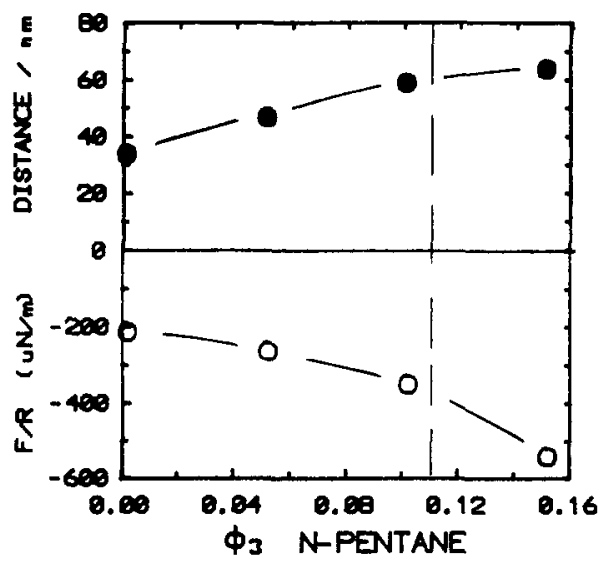

Figure 4. Maximum adhesive force (open circles) and micamica separation at the onset of detectable force (filled circles), plotted against the $n$-pentane volume fraction in the solvent mixture. The vertical bar denotes the region of solvent com. position in which FTIR-ATR experiments showed precipitation of polymer to occur.

In previous work we estimated the relative mass adsorbed from the compressed thickness at the point of steep repulsion. In the same vein, using the layer thickness plotted in Figure 3 at compression $2000 \mu \mathrm{N} / \mathrm{m}$ and assuming the density of bulk PS for simplicity, one estimates that the mass adsorbed was approximately 6 $\mathrm{mg} \mathrm{m} \mathrm{m}^{-2}$ in undiluted $\mathrm{CP}$ and $8.5 \mathrm{mg} \mathrm{m}^{-2}$ at the highest volume fraction of $n$-pentane. Obviously this estimate is an upper bound in view of solvent swelling, whose extent might depend on solvent composition. To anticipate later FTIR-ATR measurements, we note, however, that this estimate is comparable to direct measurements of the amount adsorbed onto a different substrate, silicon oxide (see below).

Also plotted in Figure 3 is the thickness at the point of maximum attractive force. This thickness increased in step with the compressed thickness and also decreased past the point of bulk precipitation.

Next we consider the effective range of surface forces (Figure 4, top) and the maximum attraction or "adhesion" (Figure 4, bottom). The former quantity increased more rapidly with the volume fraction of the nonsolvent than with that of the latter.

Both of the quantities plotted in Figure 4 increased monotonically with nonsolvent composition. Beyond the

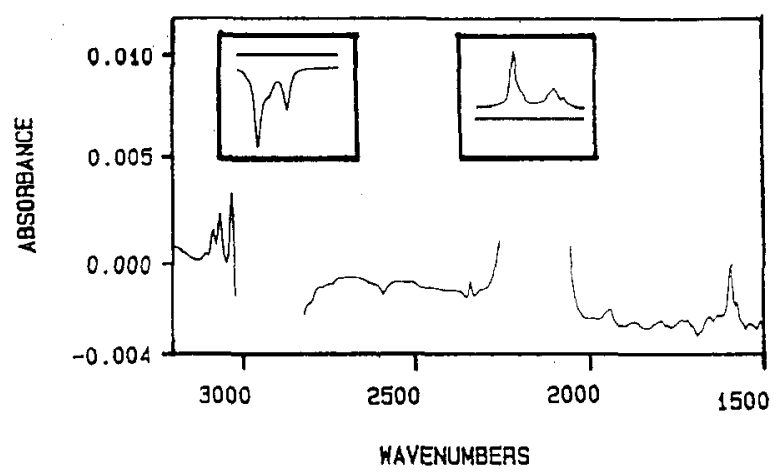

Figure 5. Infrared spectrum, measured in attenuated total reflection, of PS (concentration in solution $0.15 \mathrm{mg} \mathrm{mL}^{-1}$ ) adsorbed onto silicon oxide from $95 \%$ cyclopentane and $5 \%$ deuterio- $n$-pentane. The spectrum is a ratio to that of pure cyclopentane. Note the C-C ring bend of PS $\left(1602 \mathrm{~cm}^{-1}\right)$, from which the surface excess mass of PS was calculated. Note also $\mathrm{C}-\mathrm{H}$ stretching modes (negative peak at $2750-3000 \mathrm{~cm}^{-1}$ ), from which the negative surface excess of cyclopentane was calculated, and the C-D stretching modes $\left(2000-2300 \mathrm{~cm}^{-1}\right)$, from which the surface excess of deuterio- $n$-pentane was calculated. The $\mathrm{C}-\mathrm{H}$ and C-D stretching modes shown in the insets have absorbance scales reduced by a factor of 20 .

point of precipitation, no change is apparent either in the range of the surface forces or in the rate of increase of the adhesion: the variations in the data were smooth as the point of bulk precipitation was crossed.

These measurements in a ternary solvent system are qualitatively comparable to those obtained previously by lowering the temperature of the binary PS-CP system. ${ }^{23}$ In both instances, binary and ternary, the adhesion and the thickness of the adsorbed layers tended to increase as the quality of the solvent decreased. In seeking to understand these force and distance measurements quantitatively, it would be desirable to know the associated changes in the adsorbed amount as the proportion of nonsolvent increased and the extent of preferential swelling with the better solvent in the mixture.

From surface force measurements alone it is not possible to address these matters directly. These questions were addressed by experiments, using FTIR-ATR, of PS adsorbed onto silicon oxide under the same solvent conditions.

Representative Infrared Spectrum. Figure 5 shows a representative infrared ATR spectrum recorded for polystyrene adsorbed onto silicon oxide. Absorbance is plotted against wavenumbers. The PS $\left(M_{\mathrm{w}}=575000\right)$ was adsorbed from the concentration $0.15 \mathrm{mg} \mathrm{mL}^{-1}$ in a mixture of $95 \%$ cyclopentane and $5 \%$ deuterio- $n$-pentane. In experiments to test the increase in the adsorbed amount with variations in solvent composition, protio$n$-pentane was used, just as in the surface force experiments. In experiments to probe the extent of preferential swelling by cyclopentane, deuterio- $n$-pentane was used instead. The temperature was the same as that for the surface force experiments but was better controlled, 23.0 ${ }^{\circ} \mathrm{C}$.

The spectrum in Figure 5 is a ratio to the background spectrum of the sample cell containing pure cyclopentane. The negative peaks, in the region $2750-3000 \mathrm{~cm}^{-1}$, reflect predominantly the displacement of protiocyclopentane by $n$-pentane but also include a small positive contribution from the $\mathrm{C}-\mathrm{H}$ backbone stretching oscillations of PS. The positive peaks, in the region 2000-2300 $\mathrm{cm}^{-1}$, measure the presence of deuterio- $n$-pentane (C-D stretching oscillations). Note also the $\mathrm{C}-\mathrm{C}$ out-of-plane phenyl bend of PS (centered at $1602 \mathrm{~cm}^{-1}$ ), from which 


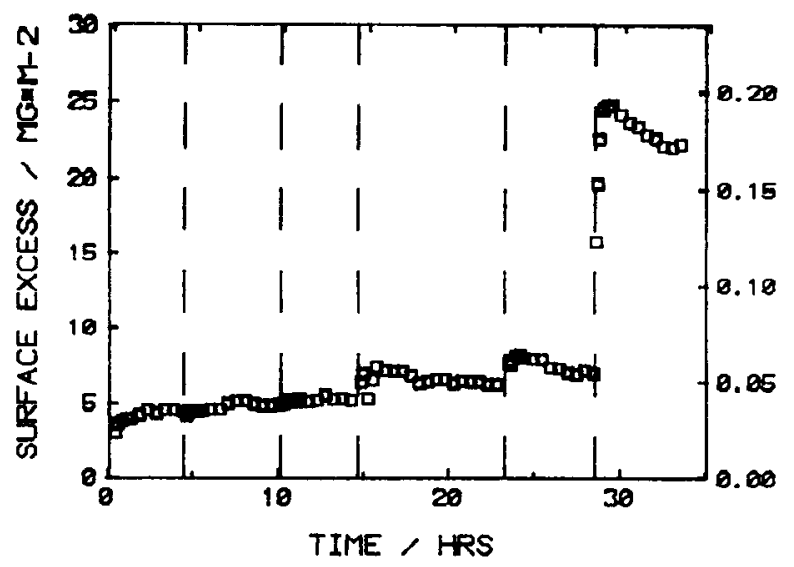

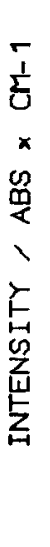

Figure 6. Surface excess mass of polystyrene $(M=490000)$ plotted against elapsed time during adsorption from mixtures of increasing $n$-pentane content. The concentration of PS in the mixtures was $0.157 \mathrm{mg} \mathrm{mL}^{-1}$. Integrated peak intensities (units: absorbance units time wavenumbers) are shown on the righthand ordinate. Dashed vertical lines indicate times at which the solution in the sample cell was replaced by a solution containing a larger fraction of protio- $n$-pentane. Steps in the data correspond to volume fractions of $n$-pentane of $0.0,0.020,0.040,0.065,0.089$, and 0.111 .

the surface excess mass of PS was calculated. The $\mathrm{C}-\mathrm{H}$ ring stretches of PS are also evident in the region 3000 $3200 \mathrm{~cm}^{-1}$. The $\mathrm{C}-\mathrm{C}$ bend was found to be more useful to quantify surface excess because the $\mathrm{C}-\mathrm{H}$ ring stretches overlapped slightly with the negative $\mathrm{C}-\mathrm{H}$ stretches of cyclopentane.

In the experiments designed to determine the extent of solvent segregation, it was convenient to obtain the spectra as a ratio to the background spectrum of PS adsorbed from cyclopentane. This eliminated the overlap of the polystyrene $\mathrm{C}-\mathrm{H}$ peaks with the negative $\mathrm{C}-\mathrm{H}$ peaks of cyclopentane.

Infrared Determination of the Adsorbed Amount onto Silicon Oxide under the Same Solvent Conditions. To track the adsorbed amount of PS, the intensity of the $\mathrm{C}-\mathrm{C}$ absorption peak centered at $1602 \mathrm{~cm}^{-1}$ was integrated after subtracting the calibrated ${ }^{30}(<1 \%)$ contribution of PS free in solution. By Beer's law, both peak height and integrated peak intensity would be proportional to the polymer concentration. However, the latter measure was more reliable in the context of the present experiment, where oscillator line shapes might be broadened by local changes in oscillator environment during the course of adsorption.

Figure 6 shows the time-dependent surface excess mass of PS measured when PS solutions $(M=490000)$ of different solvent composition were exposed to the silicon oxide surface in succession. Dashed vertical lines indicate the times when the solution in the sample cell was replaced by a solution containing additional protio- $n$-pentane. At the two highest levels of nonsolvent composition a small overshoot of the adsorbed amount is observed; this we attribute to temperature equilibration as sample was introduced to the cell at a temperature below the cell temperature. The adsorbed mass equilibrated rapidly on the time scale of hours, which lends confidence to our having achieved equilibration after waiting $8 \mathrm{~h}$ in the course of making surface force experiments.

In control experiments, the solution in the sample cell was replenished by a new PS solution of the same PS concentration. When this was done, the adsorbed mass did not increase above the level observed previously at that volume fraction of nonsolvent. Thus the possibility

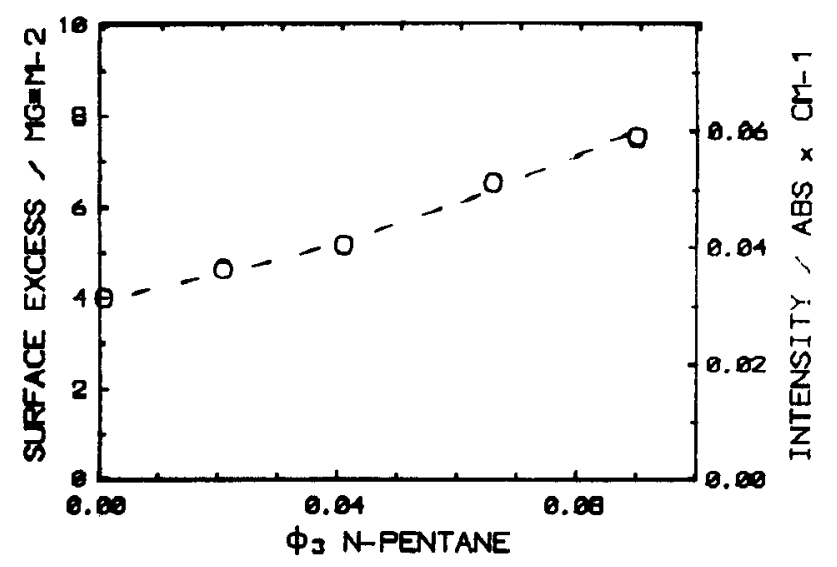

Figure 7. Equilibrated surface excess mass of polystyrene ( $M$ $=490000, c=0.15 \mathrm{mg} \mathrm{mL}^{-1}$ ) plotted as a function of the volume fraction of $n$-pentane after adsorption from mixtures of increasing $n$-pentane content.

that the enhanced adsorption we measured stemmed from fractionation to the surface of a high molecular weight tail in the polystyrene sample was ruled out.

The adsorbed mass rose monotonically up to a volume fraction of $\phi_{3}=0.089$ for $n$-pentane and then jumped abruptly at $\phi_{3}=0.111$. To observe phase separation in this range of solvent composition is consistent with the direct observations of turbidity made in the course of surface force measurements. (More detailed measurements of the bulk phase behavior would be necessary to decide if the jump in surface excess mass coincided exactly with the point of bulk phase separation.) In Figure 6, one observes that, after reaching the point of precipitation, the adsorbed mass decayed with time. This we attribute to precipitated polymer falling out of the sampling volume underneath the surface in the adsorption cell.

The equilibrated adsorption in the one-phase region of solvent compositions is plotted against the volume fraction of $n$-pentane in Figure 7. The adsorbed amount was not simply proportional to the volume fraction of nonsolvent but increased slightly more rapidly than that. A $\log -\log$ representation (not shown) demonstrates that the extra adsorption $\Delta \Gamma$ ( $\Gamma$ in addition to that measured in undiluted cyclopentane) followed by the empirical relation $\Delta \Gamma=$ $\phi_{3}{ }^{1.13}$. On the other hand, one expects that a more true representation of this increase in the surface excess would scale with proximity to the point of the phase separation. We do not attempt such an analysis at this time because the point of phase separation is not known with sufficient precision.

Testing for Surface Segregation of n-Pentane in a Mixture with Cyclopentane. We will be interested below in exploring preferential swelling of the adsorbed polymer layers by the better solvent. As a control, first experiments were performed to explore conceivable surface segregation from the binary mixture of cyclopentane and $n$-pentane without added polymer.

Because the ATR evanescent wave probes only a thin volume of solution in the vicinity of the reflection surface, solvent segregation to the surface would result in an excess of one species and a depletion of the other. The $\mathrm{C}-\mathrm{H}$ and C-D absorption peaks of protiocyclopentane and deuterio$n$-pentane are well separated in the infrared spectrum. In an extrapolation to zero $n$-pentane content, extrapolated positive infrared absorption intensity indicates surface excess and extrapolated negative absorption intensity indicates surface depletion within the volume probed by the evanescent wave. 

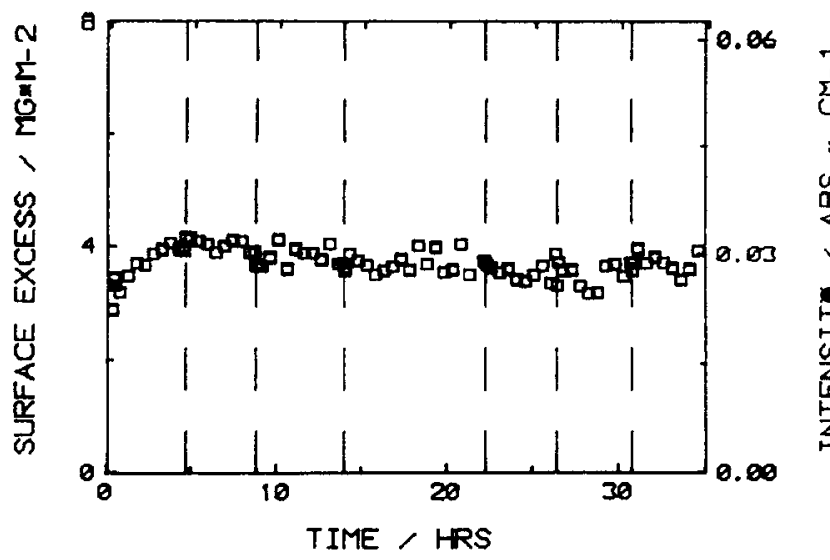

Figure 8. Surface excess mass of polystyrene $(M=575000)$ plotted against elapsed time. Initially the PS sample was allowed to adsorb from solution $\left(0.15 \mathrm{mg} \mathrm{mL}^{-1}\right)$ in cyclopentane without added $n$-pentane. Steps in the data correspond to replacing the polymer solution by mixtures of cyclopentane and deuterio- $n$ pentane, which did not contain PS. The volume fractions of $n$-pentane were 0.000 (pure cyclopentane), $0.008,0.018,0.044$, 0.061 , and 0.085 .

Figure 9 shows the extrapolation to zero content of $n$-pentane. Integrated intensity of deuterio- $n$-pentane is plotted against volume fraction deuterio- $n$-pentane in solution. The inset shows that a linear least-squares extrapolation passes through the origin within experimental uncertainty (we estimate the uncertainty as $<0.1$ $\mathrm{mg} \mathrm{m}^{-2}$ ). (The corresponding data showing cyclohexane depleted from the surface region are consistent in this finding but are not shown here, owing to larger experimental scatter.) This extrapolation to zero provides a reassuring check on the consistency in the data..$^{59}$

We had some doubt that this null result would also hold for a substrate of lower energy: $n$-pentane has a significantly lower density than cyclopentane $(0.624$ versus 0.743 $\mathrm{g} \mathrm{cm}^{-3}$ for the protio species ${ }^{60}$ ) and a correspondingly lower surface tension against air (15.7 versus $22.2 \mathrm{mN} / \mathrm{m} ; 60$ the surface tension of deuterio- $n$-pentane is expected to be similar to that of the protio species or even less to an extent up to $1 \%{ }^{61}$ ). Therefore, the silicon ATR prism was covered, using methods we have described elsewhere, ${ }^{62}$ with a close-packed monolayer of condensed octadecyltriethoxysilane (OTE), resulting in an outer layer of methyl groups. The same null result was obtained.

Test of Depletion of Nonsolvent from the Adsorbed Polymer Layer. As discussed in the Introduction, it has long been expected that a mixed-solvent system is not homogeneous; polymer coils are expected to be swollen by the better solvent and depleted in the poorer solvent. Infrared measurements in attenuated total reflection afforded the chance to measure this phenomenon directly.

Figure 8 shows the control experiment, verifying that the PS polymer did not desorb significantly as the solvent composition was changed. First, PS $(M=575000)$ was allowed to adsorb from pure cyclopentane solution, to the level $\Gamma=4.0 \mathrm{mg} \mathrm{m}^{-2}$ and then this solution was replaced by solvent mixtures, of increasing deuterio- $n$-pentane content, which did not contain polymer. The surface excess remained, throughout, at nearly $4.0 \mathrm{mg} \mathrm{m}^{-2}$ (though the decay of approximately $5-10 \%$ indicates that a small amount of desorption into the polymer-free liquid did occur over the course of over $12 \mathrm{~h}$ ). This level of desorption does not affect the results described below.

The intensity of nonsolvent ( $n$-pentane) absorption in ATR is plotted as a function of the volume fraction of the nonsolvent in Figure 9 (circles). Clearly the data are

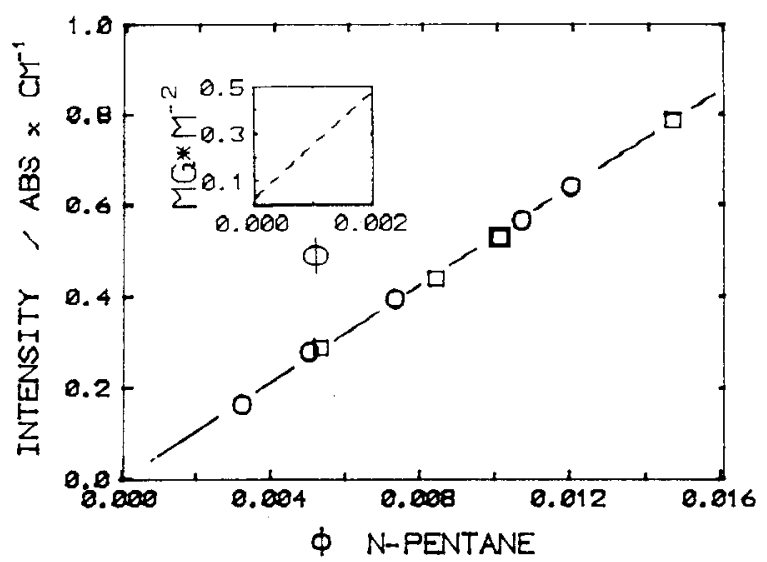

Figure 9. Test for solvent segregation. Infrared peak intensities are plotted against the fraction of deuterio- $n$-pentane in the case of binary mixtures of cyclopentane and deuterio- $n$-pentane (squares) and these same solvent mixtures in the presence of PS (circles). For the latter measurements, first, the PS $\left(M_{w}=\right.$ 575000 ) was adsorbed from cyclopentane to give the surface excess $4.0 \mathrm{mg} \mathrm{m}^{-2}$ ), and then the polymer solution was replaced by pure cyclopentane and subsequently by mixtures of cyclopentane and deuterio- $n$-pentane of the indicated compositions. The inset shows the extrapolation to the origin obtained by leastsquares fits to the data; an intercept of intensity 0.12 would correspond to $0.5 \mathrm{mg} \mathrm{m}^{-2}$ surface excess $n$-pentane. In the inset, the peak intensity is expressed in units of the surface excess mass per unit, area, which would correspond to a nonzero intercept.

indistinguishable from those obtained in the absence of adsorbed polymer (also shown in Figure 9; squares); within the experimental uncertainty the extrapolation also passes through the origin, indicating no detectable depletion $(<0.1$ $\left.\mathrm{mg} \mathrm{m} \mathrm{m}^{-2}\right) .59$

Experiments were also performed with a second polymer sample, $M_{\mathrm{w}}=3.84 \times 10^{6}$, to test the generality of these findings. In this experiment the adsorption of PS was $\Gamma$ $=5.5 \mathrm{mg} \mathrm{m}^{-2}$. This independent experiment (not shown) was consistent in showing no detectable depletion of the nonsolvent from the surface region.

Comparison of Changes in Levels of Measured Quantities as the Solvent Quality Worsened. Figure 10 compares changes in the levels of the adhesion (mica), the compressed thickness (mica), and the adsorbed mass (silicon oxide). The ratio of each quantity, relative to its value in pure cyclopentane, is plotted against the volume fraction of $n$-pentane $\left(\phi_{3}\right)$. One distinguishes plainly that all of these quantities increased in step with one another. Just before the point of precipitation, the quantities measured by surface force experiments had all increased by approximately a factor of 1.5 .

The increased adsorption of PS onto silicon oxide was even larger, a factor of 2 .

In summary, the interactions between overlapping polystyrene layers were evidently well-defined even in the two-phase solution. The range of forces and the compressed thickness leveled off or decreased slightly, while the adhesion continued to intensify smoothly as the point of precipitation was crossed.

\section{Discussion}

By using the surface force apparatus and FTIR-ATR adsorption experiments in tandem, five quantities were measured as a function of solvent composition in ternary solution. For overlapping adsorbed layers, the (1) compressed thickness, (2) effective range of interaction, (3) adhesion, and (4) thickness at maximum attraction were measured. The fifth quantity, measured by FTIR-ATR, 


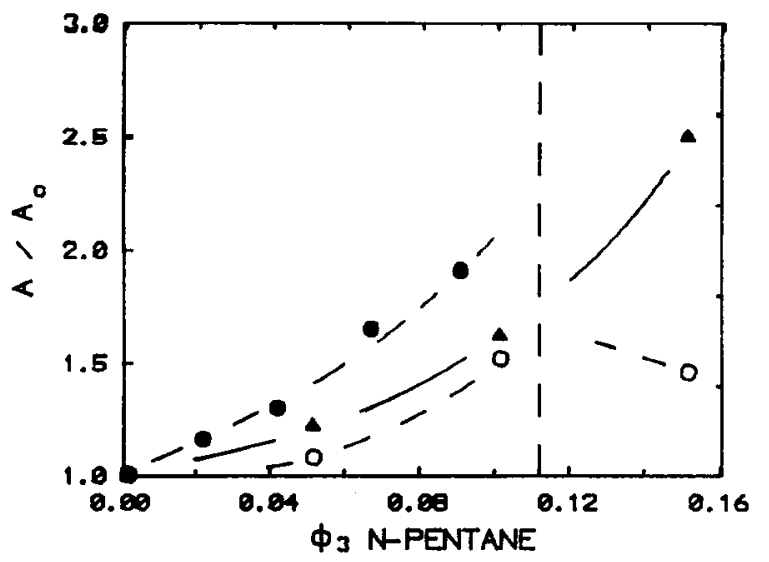

Figure 10. Comparison of surface forces and FTIR-ATR findings. Ratios of measured quantities in ternary solution, relative to their levels in undiluted cyclopentane (the ratio is designated $A / A_{0}$ ), are plotted against the volume fraction of $n$-pentane in the solvent mixture. Filled circles: surface excess mass of PS (FTIR-ATR). Filled triangles: maximum adhesive force (surface forces). Open circles: compressed thickness at $2000 \mu \mathrm{N} / \mathrm{m}$ (surface forces). The vertical bar denotes the region of solvent composition in which FTIR-ATR experiments showed precipitation of polymer to occur.

was the adsorbed mass per unit area. Our choice of solvents was intended, as discussed in the Introduction, to simplify the inherently complex ternary solvent situation to the extent possible. First, solvents were employed whose mutual enthalpic interaction was expected to be minimal. Second, it was expected that the $n$-pentane solvent, because of its similar chemical composition to that of cyclopentane, would be less prone to displace polymer from the solid-solution interface than would other nonsolvents.

The substrates were necessarily different (mica for the surface force measurements and silicon oxide for the FTIRATR measurements) although the polymer solution conditions were the same. The extent to which results on the two different substrates can reasonably be compared will be discussed below.

Possible Multilayer Adsorption near the Point of Phase Separation. The first conclusion of this study concerns the strikingly large levels of the surface excess mass observed in the FTIR-ATR experiments. While it is perhaps easy to take for granted that polymer adsorption should increase with poorer solvent quality, the large magnitude of the effect deserves emphasis. The adsorption at silicon oxide nearly doubled to $8 \mathrm{mg} \mathrm{m}^{-2}$, a noteworthy large value, before the point of phase separation in the bulk. We suggest that it is consistent with the possibility that there formed a precursor phase of phase-separated polymer, a precursor of the eventual polymer-rich bulk phase formed when the point of bulk precipitation was passed.

The customary picture of loop-train-tail conformation of adsorbed flexible homopolymers - what one might call a monomer-is well accepted. ${ }^{1}$ Polymer multilayers would not be surprising, however, on theoretical grounds. Nearly 20 years ago, Silberberg ${ }^{34}$ analyzed the case of polymer mixed in a single solvent and concluded that multilayers are feasible at temperatures within $20^{\circ}$ of the $\theta$ point. Numerical calculations of van der Schee, ${ }^{35}$ based on the Scheutjens-Fleer ${ }^{16}$ theory, reached a similar conclusion. A near-linear relation was calculated between the excess adsorbed amount (excess over the monolayer) and the logarithm of $\left[\chi-\chi_{\mathrm{cr}}\right]$ ( $\chi$ is the Flory-Huggins interaction parameter and $\chi_{c r}$ is its critical value for bulk phase separation). The magnitude of the effect predicted in those studies, a factor of $2^{34}$ or 3,35 is qualitatively consistent with our observations. In blends of two polymers, Schmidt and Binder ${ }^{36}$ and Nakanishi and Pincus ${ }^{37}$ also considered incipient wetting layers. Such an effect does not, however, appear to be yet demonstrated experimentally in either solution or the melt.

How could one decide if this effect occurred in the present experiments? We have already cited the suggestively large increase in surface excess mass. Also notable is the rapidity of the changes in the level of the surface excess when nonsolvent was added (see Figure 6). Now in order to accommodate more chains on the surface, the chains present first must rearrange so as to become physically attached by a fewer number of segments. In our view it is not at all self-evident that such large and rapid changes of the mass adsorbed could be sustained through rearrangements of loops and trains of polymer segments. On the contrary, a growing body of evidence, theoretical ${ }^{38-41}$ as well as experimental, ${ }^{30,42-47}$ suggests that such rearrangements are sluggish.

An adsorbed multilayer structure would not be surprising based on experience with mixtures of liquids of small molecules, either. As the phase-separation point is approached from the one-phase region, the asymptotic approach of multilayers, bounded in thickness, to wetting layers, of macroscopic thickness and with the properties of bulk fluid, is well studied. ${ }^{48}$ One academic instance is the thick multilayer films formed when lutidine is mixed with water. ${ }^{48}$ Of more common everyday experience is the adsorption of water films onto laboratory glassware from organic solution or even from the vapor.

If this effect occurred in the present experiments, one would expect that the mean volume fraction of the polymer in the surface region would be close to that of the polymerrich solution after the point of phase separation. A crude estimate can be made from the mica experiments if one ignores compositional gradients and orientationa ${ }^{49}$ effects. At $\phi_{3}=0.10$ the adsorption was approximately $5 \mathrm{mg} \mathrm{m}^{-2}$ and the range of forces was approximately $30 \mathrm{~nm} /$ layer, so the mean volume fraction at overlap of polymer was $\phi$ $\approx 0.2$, a reasonable ${ }^{49,50}$ estimate.

We do not attempt a more quantitative interpretation at present. If this multilayer hypothesis is correct, similar behavior would be expected to also occur in binary mixtures of polymer in a single solvent. Encouraging experiments are underway with PS dissolved in cyclohexane. Preliminary experiments show that, as the temperature is lowered in the one-phase region until the point of precipitation for different volume fractions, increases of the surface excess of similar magnitude as described above are also observed. ${ }^{63}$

Mechanisms of Interaction between Adsorbed PS Layers. The second conclusion of this study concerns interpretation of the surface force measurements. It seems that three distinctly different mechanisms, peculiar to mixed-solvent systems, may underlie the interactions between adsorbed polymer layers in mixed solvents.

First, competitive adsorption with a solvent that adsorbs more strongly than a polymer segment (a "displacer" solvent) will result in a lesser adsorbed amount and consequently in variations in the layer thickness. It was attempted to avoid this complication, to the extent possible, in the present study by choosing solvents of similar chemical composition.

Second, interactions stemming from solvent-solvent interactions can come into play. There are two limits to consider: extremely unfavorable enthalpy of mixing between the two solvent species ${ }^{15}$ and the situation if the two solvents are close to their critical temperature. ${ }^{55} \mathrm{We}$ have also sought to avoid these complications. 
Third, the mechanism addressed in this study, is the effect of changing the solvent quality. This is expected to influence, to the extent that they can be discussed separately, both the surface excess and the forces between overlapping layers. We have provided direct measurements that confirm the view that the surface excess will be greater in a poorer solvent, while other factors are held constant.

When adsorbed polymer layers begin to overlap as they are brought closer together, the segmental concentration between the surfaces is augmented. It has become customary to analyze the resulting forces in terms of osmotic pressure interactions and bridging interactions. ${ }^{1}$ In the work reported the bridging effect should have been increasingly impeded at high levels of surface excess mass, and thus it seems an unlikely origin of the increased adhesion associated with high levels of surface excess mass.

In analyzing force-distance data, one customarily presumes that the $T_{\theta}$ is presumed to be well-defined and unchanged near a surface. ${ }^{1}$ It then follows, in binary solution, that those forces between overlapping layers of osmotic origin are repulsive above the $\theta$ temperature and attractive below it, as the second virial coefficient changes from positive to negative. ${ }^{1}$ Comparable effects may be expected to occur in mixed solvents. One may broadly expect to find large attraction for osmotic reasons when the mixed solvent contained a large proportion of nonsolvent, as has been discussed. ${ }^{24-26}$ This interpretation is not without ambiguity in the context of the present experiments, however, as discussed below.

Considerations of Interfacial Energy in Surface Force Measurements. Continuous Transition of the Interfacial Forces, but Not of the Surface Excess Mass, between Single-Phase and Two-Phase Solutions. Past the point of bulk phase separation the polymer is distributed between three phases: polymer in solution (polymer-rich), polymer in solution (polymerpoor), and the phase of adsorbed polymer. We suggested above that the layer of adsorbed polymer can grow to be a precursor of the polymer-rich solution phase. In principle, one might have argued that this should not be so: the polymer-rich phase would not nucleate at the surface because the effective volume fraction of the surface phase would be larger than of the polymer-rich phase..$^{49}$ A discontinuous jump in the surface excess mass of PS was, however, observed (Figure 6).

A noteworthy feature of the data in Figures 3,4 , and 10 , commented upon already above, is the continuous changes observed in all of the surface force measurements as homogeneous bulk solution became phase-separated bulk solution. In contrast, the surface excess mass measured by FTIR-ATR jumped discontinuously past the cloud point. It would be conceivable but farfetched to write off the discrepancy to different adsorption behavior onto silicon oxide and onto muscovite mica. More likely is that surface forces do not change in step with changes in the surface excess mass. The continuous transition in forces measured, as the bulk solution crossed from homogeneous to two phases, suggests to us that the origin of the forces did not change character either. A possible explanation of the continuous change in adhesion lies in considerations of interfacial energy.

Interfaces are created (one interface at each surface) as adsorbed polymer layers are separated in the two-phase region. The interfaces are between adsorbed polymer (a polymer-rich phase) and polymer coexisting in phaseseparated solution. In the present experiments at the highest proportions of nonsolvent (highest surface cov- erages), the extent of bridging of chains between the two surfaces is expected to have been minor compared to its extent at lower proportions of nonsolvent (lower surface coverages) and therefore cannot account for this large adhesion in excess of that measured in the absence of nonsolvent. As for the osmotic effects, for which it is customary to consider as being the second possible source of interaction between overlapping polymers layers, ${ }^{1}$ in the twophase region, these are an element of the interfacial energy.

Quantitatively, the adhesion of $-600 \mu \mathrm{N} / \mathrm{m}$ measured at $\phi_{3}=0.15$ corresponds, using the Derjaguin approximation $W(D)=F(D) / 2 \pi R,{ }^{33}$ to the energy $W(D)=1 \times$ $10^{-4} \mathrm{~J} / \mathrm{m}^{2}$ necessary to create two interfaces. Thus we reach the (upper bound) estimate for interfacial tension of $\gamma \approx 0.05 \mathrm{~mJ} \mathrm{~m}^{-2}$. This is not strictly comparable to the interfacial tension between coexisting phases in bulk solution because it involves the interface between adsorbed polymer and solution. Nonetheless, this estimate is in general agreement with theoretical expectations 49-52 and $^{-5}$ the few experiments ${ }^{53,54}$ regarding the interfacial tension between coexisting phases in bulk solution.

Parenthetically, we note that attractive forces comparable in magnitude to those measured in the present experiments were also reported in surface force experiments between layers of PS near the two-phase region in binary solution (PS adsorbed from solution in cyclohexane). ${ }^{1}$ It has become apparent that no theory yet predicts the levels of the minima of those profiles measured in binary solution. As Ingersent, Klein, and Pincus pointed out in ref 56, a possible origin of the discrepancy was their use a virial expansion to describe the osmotic interactions and consequent restriction of interactions to low volume fractions of polymer. Whatever the reason, the predicted values are too low by a factor of $2-3,1,54,55$

As already noted above, the rate of increase of the adhesion between overlapping layers did not change character as the bulk solution crossed from homogeneous to two phases (cf. Figure 4). Without wishing to be overly speculative, we suggest tentatively that the origin of the above discrepancy between theory and experiment might lie in underestimating the interfacial tension between adsorbed polymer layers and the polymer solution adjacent to it.

Tests of Depletion of Nonsolvent from Adsorbed PS Layers. These experiments have allowed a direct test of the expectation that the better solvent will preferentially swell polymer coils in a ternary solution. The effect, if present, was too small to be detected. Interpretation of this null result is not attempted at this time.

\section{Concluding Remarks}

The main controlling variables in polymer adsorption are solvent quality and the intensity of interaction with the substrate. These experiments have investigated the influence on adsorption of ternary solvent composition as nonsolvent was added to the point of phase separation. In general this would not be a well-posed question: interactions of the substrate surface with the nonsolvent may be as important as the solvent quality, and strong substrate-nonsolvent interaction will decrease the adsorption of the polymer. The cyclopentane/ $n$-pentane solvent system was chosen for its intended simplicity in this respect and also for its expected athermal mixing. We emphasize the systematic nature of the quantitative changes described above.

It is clear that the level of surface excess mass is sensitive to many subtle parameters. Although this paper has emphasized the effect of nonsolvent, it is perhaps worthwhile to note that, even from binary mixtures of PS and 
cyclopentane, the levels of surface excess found in this study are about twice as large as observed for the adsorption from cyclohexane onto mica ${ }^{24-26}$ or onto silica particles. ${ }^{58}$ Evidently, it is not meaningful to ask, independent of the particular system, how much polymer will adsorb, even though the solvent quality conditions may nominally be the same.

A question for future research that is raised by this study concerns the solvent composition within an adsorbed polymer layer in ternary solution. The present conclusion of no preferential depletion of the poorer solvent is probably not a general one and may be expected to depend on the chemistry of each particular system. For example, in mixed solvents involving heptane $e^{24}$ and acetone, ${ }^{25}$ the chemistry suggests that (unlike the present system) preferential adsorption of these nonsolvents should be pronounced. This in turn suggests that solvent composition may be extremely inhomogeneous near a surface: enhanced in the poorer solvent directly at the solid surface and (owing to swelling of the polymer coils by the better solvent) enhanced in the other solvent at distances farther removed but still located within the adsorbed coils.

The second question raised by this study concerns the influence of interfacial energy on surface forces. When overlapping layers are separated in the two-phase region, the adhesive minimum of a force-distance profile measures the interfacial tension between the polymer-rich adsorbed layer and the polymer-poor solution. We have argued that also in one-phase systems the energy to create new interfaces may contribute to the adhesion. The possibility of quantifying questions of polymer interfacial energy in solution using surface force measurements warrants further investigation.

The third question for future work concerns the possibility of multilayers. We have argued that the large increase in surface excess mass of polymer, observed near the point of phase separation, may reflect the presence of polymer multilayers with a thickness such that, in addition to the loops and trains of chains adsorbed to the surface, they may contain chains none of whose segments are located physically at the solid surface. These multilayers would be precursors of the eventual polymer-rich bulk phase, which forms in the two-phase region. Measurements of the extent of adsorption without direct information on the associated structure cannot, however, yield clear pictures of the resulting interfacial structure. With other techniques, such as neutron reflectivity or evanescent waves of shorter wavelength, it would be possible to study the structure and density profile of such multilayers directly.

Acknowledgment. This work was supported by the National Science Foundation (Polymers Program), Grant DMR-87-18136. H.-W.H. acknowledges support from the NSF, Grant DMR-89-20538. We thank I. Szleifer for a discussion and Jack Douglas for comments.

\section{References and Notes}

(1) For a review see: Patel, S. J.; Tirrell, M. Annu. Rev. Phys. Chem. 1989, 40, 597.

(2) Tompa, H. Trans. Faraday Soc. 1949, 45, 1142.

(3) Scott, R. L. J. Chem. Phys. 1949, 17, 268.

(4) Flory, P. J. Principles of Polymer Chemistry; Cornell University Press: Ithaca, NY, 1953

(5) Shultz, A.; Flory, P. J. J. Polym. Sci. 1955, 15, 231.

(6) Yamakawa, H. J. Chem. Phys. 1967, 46, 973.

(7) Dondos, A.; Benoit, H. J. Polym. Sci., Part B 1969, 7, 335.

(8) Dondos, A.; Izumi, Y. Makromol. Chem. 1980, 181, 701.

(9) Nakata, M.; Numsawa, N. Macromolecules 1985, 18, 1736.

(10) Kappeler, C.; Schafer, L. macromolecules 1990, 23, 2766. Tanaka, F.; Ushiki, H. Macromolecules 1988, 21, 1041.
(11) Majda, J. J.; Fredrickson, G. H.; Larson, R. G.; Helfand, E. Macromolecules 1988, 21,726.

(12) Tejero, R.; Gómez, C.; Celda, B.; Gavara, R.; Campos, A. Makromal. Chem. 1988, 189, 1643.

(13) Celda, B.; Gavara, R.; Gómez, C.; Tejero, R.; Campos, A. Makromol. Chem. 1988, 189, 1657.

(14) Viras, F.; Viras, K. J. Polym. Sci., Part B. 1988, 26, 2525.

(15) Wolf, B. A.; Blaum, G. J. Polym. Sci., Polym. Phys. Ed. 1975, 13,1115 .

(16) Fleer, G.; Lyklema, J. In Adsorption from Solution at the SolidLiquid Interface; Parfitt, G. D., Rochester, C. H., Eds.; Academic Press: New York, 1983.

(17) de Gennes, P.-G. Macromolecules 1981, 14, 1637.

(18) Snyder, L. R. Principles of Adsorption Chromatography; Marcel Dekker: New York, 1968.

(19) Glöckner, G. J. Polym. Sci., Polym. Symp. 1980, 68, 179.

(20) Cohen-Stuart, M. A.; Fleer, G. J.; Scheutjens, J. M. H. M. J. Colloid Interface Sci. 1984, 97, 515.

(21) van der Beek, G. P.; Cohen-Stuart, M. A.; Fleer, G. J.; Hofman, J. E. Langmuir 1989, 5, 1180.

(22) Hu, H.-W.; Van Alsten, J.; Granick, S. Langmuir 1989, 5, 270.

(23) Hu, H.-W.; Granick, S. Macromolecules 1990, 23, 613.

(24) Marra, J.; Hair, M. L. Macromolecules 1988, 21, 2349.

(25) Marra, J.; Hair, M. L. Macromolecules 1988, 21, 2356.

(26) Marra, J.; Christenson, H. K. J. Phys. Chem. 1989, 93, 7180.

(27) Saeki, S.; Kuwahara, N.; Konno, S.; Kaneko, M. Macromolecules $1973,6,589$.

(28) Barry, G. C.; Casassa, E. F.; Liu, P.-Y. J. Polym. Sci., Polym. Phys. Ed. 1987, 25, 673.

(29) McGlinn, T. C.; Kuzmenka, D. J.; Granick, S. Phys. Rev. Lett. $1988,60,805$.

(30) Johnson, H. E.; Grantick, S. Macromolecules 1990, $23,3367$.

(31) Deleted in Revision.

(32) Harrick, N. J. Internal Reflection Spectroscopy; Interscience: New York, 1967.

(33) Israelachvili, J. N. Intermolecular and Surface Forces; Wiley: New York, 1985.

(34) Silberberg, A. J. Colloid Interface Sci. 1972, 38, 217.

(35) van der Schee, H. A. Ph.D. Thesis, Wageningen, Netherlands, 1984.

(36) Schmidt, I.; Binder, K. J. Phys. (Paris) 1985, 46, 1631.

(37) Nakanishi, H.; Pincus, P. J. Chem. Phys. 1983, 79, 997.

(38) de Gennes, P.-G. in New Trends in Physics and Physical Chemistry of Polymers; Lee, L.-H., Ed.; Plenum: New York, 1990.

(39) de Gennes, P.-G. C.R. Acad.Sci. (Paris) 1988, II 306, 183; 1988, II 306, 739.

(40) de Gennes, P.-G. C. R. Seances Acad. Sci. 1985, 301, 1399.

(41) Ligoure, C.; Leibler, L. J. Phys. (Paris), in press.

(42) Pefferkorn, E.; Carroy, A.; Varoqui, R. J. Polym. Sci., Polym. Phys. Ed. 1985, 23, 1997.

(43) Pefferkorn, E.; Haouam, A.; Varoqui, R. Macromolecules 1988, $21,2111$.

(44) Pefferkorn, E.; Haouam, A.; Varoqui, R. Macromolecules 1989, $22,2677$.

(45) Haouam, A.; Pefferkorn, E. Colloids Surf. 1988/1989, 34, 371.

(46) Kuzmenka, D. J.; Granick, S. Colloids Surf. 1988, 31, 105.

(47) Char, K.; Gast, A. P.; Frank, C. W. Langmuir 1988, 4, 989.

(48) For a review see: Dietrich, S. Phase Transitions Crit. Phenom. $1988,12,1$.

(49) Szleifer, I., private communication.

(50) Szleifer, I.; Widom, B. J. Chem. Phys. 1989, 90, 7524.

(51) Nose, T. Polym. J. 1976, 8, 96.

(52) Vrij, A.; Roebersen, G. J. J. Polym. Sci., Polym. Phys. Ed. 1977, $15,109$.

(53) Dobashi, T.; Nakata, M.; Kaneko, M. J. Chem. Phys. 1980, 72, 6685.

(54) Shinozaki, K.; van Tan, T.; Saito, Y.; Nose, T. Polymer 1982, 23,728 .

(55) de Gennes, P.-G. J. Phys. (Paris) 1976, 37, L-59.

(56) Ingersent, K.; Klein, J.; Pincus, P. Macromolecules 1990, 23, 548.

(57) Fleer, G. J.; Scheutjens, J. M. H. M. J. Colloid Interface Sci. 1986, 111, 504 .

(58) Vander Linden, C.; Van Leemput, R. J. Colloid Interface Sci. $1978,67,48$.

(59) The infrared spectra described here were obtained by using $2-\mathrm{cm}^{-1}$ resolution. In an earlier work, we obtained misleading results using lower resolution $\left(8 \mathrm{~cm}^{-1}\right)$.

(60) Riddick, J. A.; Bunger, W. B.; Sakano, T. K. Organic Solvents: Physical Properties and Methods of Purification, 4th ed.; John Wiley \& Sons: New York, 1986.

(61) Bartell, L. S.; Roskos, R. R. J. Chem. Phys. 1966, 4, 457.

(62) Kessel, C. R.; Granick, S. Langmuir, in press.

(63) Johnson, H. E.; Granick, S., Macromolecules, in press. 